\title{
Dissociation of conditioned emotional and avoidance responses due to ECS: A cautionary note'
}

\author{
BRENDAN A. MAHER, UNIVERSITY OF WISCONSIN
} DONALD J. LEWIS, RUTGERS UNIVERSITY

Because of the measures of conditioned emotional response (CER) used by Gerbrandt (1965) and certain other methodological difficulties, his results are not considered crucial to the hypothesis that the impairment by ECS of previously learned responses may be due to some competing response conditioned to cues associated with the ECS coma.

As there are several errors in Gerbrandt's recent paper in this journal (1965), it is necessary for the sake of the reader that they be corrected. Some are simply errors in reporting; others are more germane to the issue of his study.

Maher \& McIntire (1960)-McIntire was misspelleddid find that lesions of the frontal area affected the locomotor component of the CER, leaving the defecation component unchanged. Frontal lesions abolished the tense motor "freezing" response-the animals became very active and mobile after the lesions. Gerbrandt apparently was using the term CER in a few places to mean simply defecation and urination in the face of threat. This is, of course, not the generally accepted meaning of the term; rather the freezing response is the component of the fear response that has usually been referred to as the CER and the one we had in mind in proposing our hypothesis. On the other hand, he goes on to state that the CER has been reported as having a suppressant effect on operant behavior-a difficult situation to envisage if the CER is defined purely in excretory terms. Of course, the suppression of ongoing motor responses is the most customary operational definition of the CER, and it is this suppression of locomotion that did disappear in the Maher and McIntire study. In brief, the Maher and McIntire study was reported inaccurately, and a new definition of CER was made without being clear and consistent about it.

In the light of this it is difficult to know how to evaluate the comment about the avoidance deficit found in frontal animals by Thompson (1964), a difficulty that is compounded by the lack of any citation of this study in the References. 2

However, Gerbrandt's ambiguity about the meaning of CER seems unimportant to his own study. In his investigation it is clear that he refers only to defecation and urination. He feels that the disssociation reported between this pattern of response and avoidance responding is "crucial in determining the validity of the recent hypothesis of ECS effects advanced by Lewis \& Maher (1965)." (The citation of this hypothesis was incomplete and erroneously attributed to thePsychological Bulletin. The relevant article appeared in the Psychological Review and is cited correctly at the end of this paper.) Gerbrandt opposes our hypothesis of conditioned inhibition to the hypothesis that ECS produces retrograde amnesia. We do not, of course, dispute that ECS produces impairment in the performance of previously learned responses. What is at issue is whether this impairment is to be ascribed to engram destruction, or whether it is due to the interference of some competing response (of which conditioned inhibition is just one possibility).

Nor is there any intention to deny that memory has a physical basis in neural tissue and is therefore, presumably, frangible under some circumstances. What we are interested in is explaining the large bulk of data on behavioral deficit following ECS in the many studies in which engram destruction was not a plausible alternative, e. g., those involving proactive effects, multiple convulsions, etc.

Our inhibition hypothesis suggests that the cues immediately present and specifically associated with the onset of ECS-produced coma might become conditioned stimuli for sub-comatose behavior, i. e., for behavior associated with low levels of arousal. In discussing this possibility we noted that Williams (1961) had pointed out an interesting paradox of electroconvulsive shock: "She noted that following ECS, the treated animals when in their home cages huddled in a ball in the rear corners of the cages or crouched on the food containers. They were hyperirritable when handled and squealed, leaped or froze when $\mathrm{E}$ attempted to lift them" ! This behavior was in marked contrast to their response when faced with the experimental apparatus in which a CER had been established earlier. "In this experiment, the ECS animals behaved as if the grid were not emotion arousing" (Lewis \& Maher, 1965, p. 235). This is an excellent description of the state of affairs we have in mind. Others have made similar reports of lowered emotionality following ECS (e. g., Page, 1941).

Gerbrandt appears to have measured (i. e., assigned points for) defecation and urination while the rat was 'being carried from its home cage, and being introduced into the avoidance apparatus with ear-clips attached." On the other hand he has measured "avoidance" in terms of the animal's behavior while on the safe platform. As he has failed to indicate how many of the emotional responses (defecation and urination) were produced during handling en route from cage to appara- 
tus and before the moment of being placed on the platform, it is not possible to know what his data represent at all! The key stimulus situation is obviously the one that exists for the animal on the platform, not the complex of stimuli associated with being handled, lifted out of a cage, etc. In most laboratories this kind of stimulation has been paired with many other random events and would be difficult to establish as a CS for a response that was to come in an apparatus some time later. If this kind of handling en route to an experimental apparatus were likely to become a CS for the specific responses that are to be elicited in the apparatus, the conduct of conditioning experiments would be very difficult indeed!

His data regarding the avoidance responses are, on the contrary, pertinent to our hypothesis, dealing as they do with behavior that is under the stimulus control of features of the experimental apparatus. Here we see that the effects of ECS appear to include an absence of the tense crouching that is produced by footshock. Just as we predict, fear motivated crouching has not developed although we cannot tell whether the ECS rats are less aroused than control rats for there are no data to provide us with a base rate for stepping off or staying

\section{Reply to Maher and Lewis by Lauren K. Gerbrandt}

In publishing my paper in this journal, I intended to show that the Lewis and Maher hypothesis (1965) is based on the assumption that changes in the CER are a necessary condition for changes in the occurrence of avoidance, and that evidence to date does not bear out such an assumption. I pointed out that although some studies have shown correlations between freezing, response suppression, urination-defecation and other indicants of the CER and avoidance performance, Kamin, Brimer, \& Black (1963) have clearly shown that suppression effects of the CER are routinely le s sened while animals were becoming maximal in avoidance performance, whereas declining avoidance performance during extinction was not accompanied by decreases in suppression effects. Since changes in the CER do not seem to be a necessary condition for changes in the occurrence of avoidance, their presence or lack as affected by ECS was not deemed theoretically relevant to changes in avoidance. I simply stressed the possibility that my data were just a small part of the vast literature accruing (Thompson, Lesse, \& Rich, 1963; Kamin, Brimer, \& Black, 1963; Mikulus \& Isaacson, 1965) which points to the same conclusion. Since Thompson, Lesse, \& Rich (1963) found conditioned emotional responses such as squealing and abrupt changes in respiratory rate independent of the conventional freezing indicator used by Maher and Lewis, it was tentatively concluded that a necessary relation also does not exist between activity and the CER and the other CER effects. Since I had found a linear relationship between urination-defecation and avoidance on the platform by unshocked, unpunished animals. Under the circumstances, therefore, we must point out that the hypothesis that we have advanced is not tested by this study, the results of which are hardly "crucial" in determining its fate. Coming as they do, from an ECS study that uses multiple treatments, they are, of course, irrelevant to consolidation theory also.

\section{References}

Gerbrandt, L. K. Dissociation of conditioned emotional and avoidance responses due to ECS. Psychon. Sci., 1965, 2, 385-386.

Lewis, D. J., \& Maher, B. A. Neural consolidation and electroconvulsive shock. Psychol. Rev., 1965, 72, 225-239.

Maher, B. A., \& McIntire, R. W. The extinction of the CER following frontal ablation. J. comp. physiol. Psychol., 1960, 53 549-552.

Page, J. D. Studies in electrically induced convulsions in animals. J. comp. physiol. Psychol., 1941, 31, 181-194.

Williams, G. J. The effect of electroconvulsive shock on an instrumental conditioned emotional response ("conflict"). $J$. comp. physiol. Psychol., 1961, 54, 633-637.

\section{Notes}

1. This paper was prepared with the support of Grant No. MH 07129 of the National Institutes of Health.

2. Because of this, we cannot cite it in the references either.

performance in the footshock group of a previously published study (Gerbrandt \& Thomson, 1964) it was decided to replicate this portion of the study in comparison with ECS punishment. The group of animals given ECS as punishment again showed very little avoidance of ECS, although their emotionality was easily chained back by conditioning (even after a single ECS) to the cues of picking up the animal from his home cage for daily trials. Contrary to the subjective evidence of Williams (1961), while in the apparatus these subjects would often squeal and tremor. The apparatus was particularly bothersome to clean after a day's "run" with these animals. It is surprising, even though theoretically irrelevant, that Maher and Lewis question the emotionality of these animals. Because of limitations in article length in this journal, it was withheld that $75 \%$ of these rats developed severe stomach ulceration (4 or more lesions larger than $2 \mathrm{~mm}$ ) as a result of ECS administration. Perhaps Maher \& Lewis should clarify these interesting ramifications of "relaxation"! The ad hoc referral to "absence of tense crouching" in the performance of these animals might better be termed "an absence in memory of which response was punished." Deficits in recall due to ECS have been reported as well in humans (Williams, 1950). Humans have also shown evidence of increases in emotionality due to threat of impending ECS treatments (Kast \& Zweibel, 1954). These CER effects were greatest when patients were in the place of ECS delivery.

If we are to consider viable the Lewis and Maher

Continued on page 198 . 